\title{
Chemi-ionization processes in Narrow-Line Seyfert 1 Galaxies
}

\section{Milan S. Dimitrijević*}

Astronomical Observatory, Volgina 7, 11060 Belgrade, Serbia,

LERMA, Observatoire de Paris, UMR CNRS 8112, UPMC, 92195 Meudon Cedex,

France

E-mail: mdimitrijevicdaob.rs

\section{Vladimir A. Srećković and Ljubinko M. Ignjatović}

Institute of Physics, Belgrade University, Pregrevica 118, 11080 Zemun, Belgrade, Serbia E-mail: vladadipb.ac.rs, Ijubadipb.ac.rs

In this work, the chemi-ionization/recombination processes in atom- Rydberg atom collisions are considered as factors of influence on the ionization degree, atom excited-state populations and hydrogen molecule abundance in the dense moderately ionized regions in AGNs, as for example in clouds around the central black hole. The obtained results demonstrate the fact that the considered chemi-ionization/recombination processes for principal quantum number $2 \leq n \leq 10$, and $5000 \mathrm{~K} \leq T \leq 12000 \mathrm{~K}$ must have a certain influence on the optical properties of some parts of Narrow-Line Seyfert 1 Galaxies.

Revisiting narrow-line Seyfert 1 galaxies and their place in the Universe - NLS1-2018 9-13 April 2018

Padova Botanical Garden, Italy

*Speaker. 


\section{Introduction}

For diagnostic methods needed in order to estimate physical conditions in the broad line region (BLR) in the narrow-line Seyfert 1 galaxies NLS1 the study of the influence of various atomic and molecular processes may be of interest. In [प], among 18 different collisional reactions, the associative chemi-ionization is included in the investigation of hydrogen molecule formation in quasar BLR. For this process, conditions in dense weakly ionized parts of BLR clouds should be favorable. It is interesting that [Z], among possible explanations for the rapid X-ray variability from the irradiated surface of BLR clouds in NLS1, quotes "the existence of very compact emitting region". Since the influence of the mentioned chemi-ionization/recombination processes is very sensitive to the change of density, this may be used to investigate if such compact regions exist in BLR clouds in NLS1. Consequently, it is of interest to investigate the influence of chemi ionization/recombination processes in similar conditions that probably hold in some parts of BLR clouds in NLS1

When an electron is excited into a high lying Rydberg state, with large principal quantum number, even inelastic thermal collisions can be sufficiently energetic to lead to ionization reactions [ [ $[$, 目]. These types of reactions can be classified as chemi-ionization reactions (associative ionization and non-associative ionization),

$$
\begin{gathered}
A^{*}(n)+A \Leftrightarrow A_{2}^{+}+e, \\
A^{*}(n)+A \Leftrightarrow A+A^{+}+e,
\end{gathered}
$$

where $A$ and $A^{+}$are atoms and atomic ions in their ground states, $A^{*}(n)$ is an atom in a highly excited (Rydberg) state with the principal quantum number $n \geq 2$.

The chemi-ionization processes and production of Rydberg atoms i.e., chemi-recombination are of importance in cool dwarf stars and, especially, cool white dwarfs [ [G, [6]. It is pointed out that the Rydberg states that arise have large dipole moments, leading to strong absorption in the infrared, and also to the appearance of polarization [ [ $]$ ]. It should also be noted that the processes $(\mathbb{[}, \mathbb{C})$ and $(\mathbb{[} \cdot \mathbf{Z})$ are very important for modelling the moderately

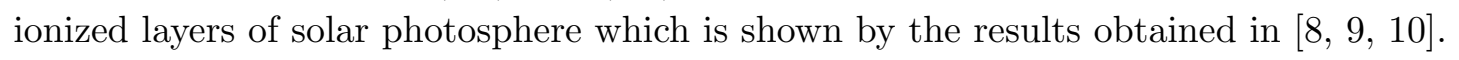

Analyzing literature related to atomic and molecular processes important for physics and chemistry of AGN we found only very poor and unreliable data needed for modelling. The available data is of questionable quality, and different prescriptions for the rate coefficients give significantly different results. This has encouraged us all to continue further research. In this paper one of our main aims is to draw attention to the importance of

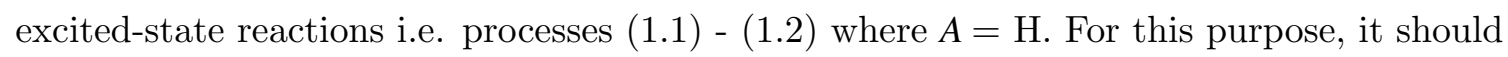
be demonstrated that in some parts of Narrow-Line Seyfert 1 (NLS1) galaxies these processes are factors of influence on the ionization degree, atom excited-state populations and hydrogen molecule formation for ranges of parameters which are relevant to the existing conditions.

\section{THE CALCULATED QUANTITIES}

The partial cross sections $\sigma_{c i}^{(a, b)}(n ; E)$ of the chemi-ionization processes $(\mathbb{L} \mathbb{\mathbb { Z }})$ and $(\mathbb{L} \boldsymbol{Z})$ 
i.e. associative and non-associative ionization channel are determined as in [0, ㅁ] . Taking cross sections $\sigma_{c i}^{(a, b)}(n ; E)$, the corresponding partial rate coefficients $K_{c i}^{(a, b)}(n ; T)$ of the chemi-ionization processes $(\mathbb{\square} \mathbb{C})$ and $(\mathbb{\square})$ are obtained. The partial rate coefficients of the inverse chemi-recombination processes with $K_{c r}^{(a, b)}(n ; T)$ are obtained from the principle of thermodynamical balance. Using these partial rate coefficients we determined the total

$$
K_{c i, c r}(n, T)=K_{c i, c r}^{(a)}(n, T)+K_{c i, c r}^{(b)}(n, T) .
$$

These rate coefficients enable inclusion of these excited-state reactions in modelling of some regions in NLS1 galaxies.

We will consider processes $(\mathbb{\square}$ ) and (마) with $A=\mathrm{H}$ within the regions $5 \leq n \leq 10$ and

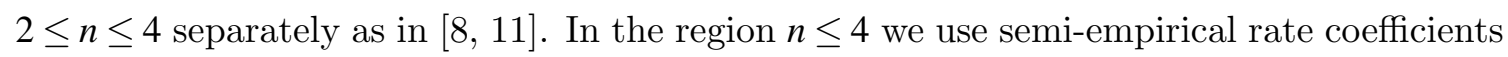
and for $n \geq 5$ we determine the rate coefficients following the method from [ $[8]$. Using rate

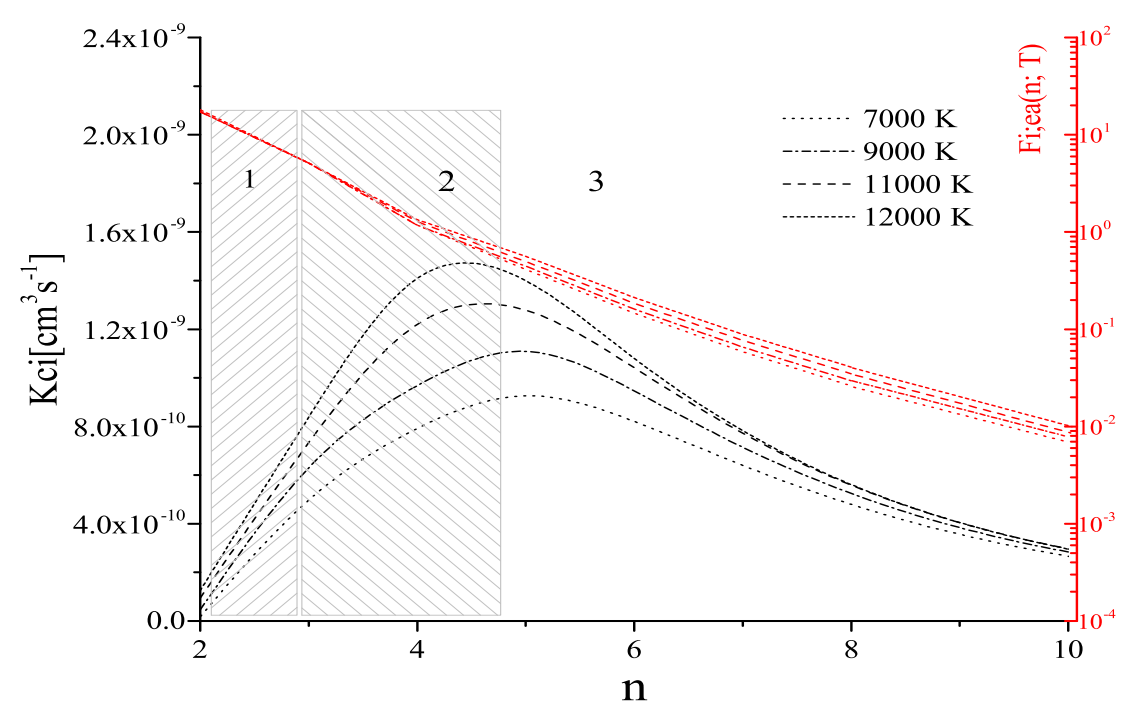

Figure 1: Values of $F_{i, e a}=I_{c i} / I_{i ; e a}$, for a density of $10^{13} \mathrm{~cm}^{-3}$, and of $K_{c i}\left[\mathrm{~cm}^{3} / \mathrm{s}\right]$ (एत) as a function of $n$ and $T$. The shaded areas schematically present the region of importance in flux analyses (ए2).

coefficients we can obtain the total chemi-ionization and chemi-recombination fluxes caused by the processes $(\mathbb{\square} \mathbb{C})$ and $(\mathbb{\square} 2)$ i.e.,

$$
I_{c i}(n, T)=K_{c i}(n, T) \cdot N_{n} N_{1}, \quad I_{c r}(n, T)=K_{c r}(n, T) \cdot N_{1} N_{i} N_{e} .
$$

Using these expressions, we can calculate quantities $F_{i, e a}(n, T)=I_{c i}(n, T) / I_{i, e a}(n, T)$ which characterize the relative efficiency of partial chemi-ionization processes $(\mathbb{\square}$ ) and (마) together and the impact electron-atom ionization $I_{i ; e a}$ as one of the most important concurrent processes in the considered plasma.

\section{RESULTS AND DISCUSSION}

The values of the calculated total chemi-ionization and recombination rate coefficients $K_{c i}(n, T)$ and $K_{c r}(n, T)$ are illustrated in Fig. $\mathbf{\square}$. The results cover the regions of $2 \leq n \leq 10$ 
and $5000 \mathrm{~K} \leq T \leq 12000 \mathrm{~K}$ which are relevant for considered cases. This will enable the correct inclusion of these processes in the modelling of weakly ionized regions of NLS1s, where density is sufficiently high. We can see in Fig. $\square$ that for a density of $10^{13} \mathrm{~cm}^{-3}, F_{i, e a}$ values of around 10 show that the efficiency of the investigated chemi-ionization processes is ten times larger than that of the concurrent impact electron-atom ionization. The val-

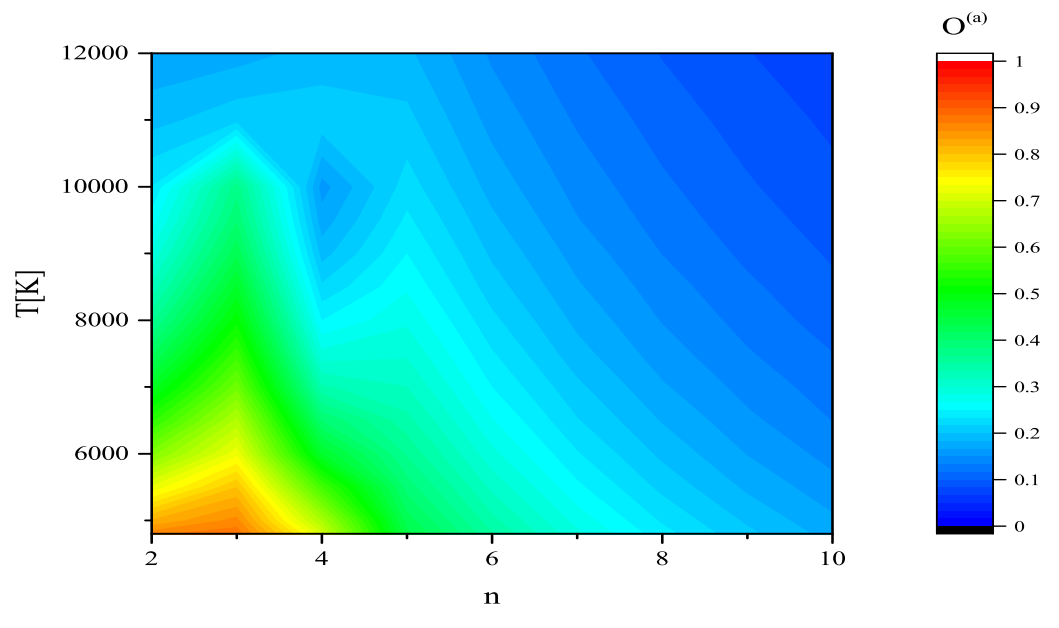

Figure 2: Relative contribution of associative channels $(\square \mathbb{\square})$ of chemi-ionization/recombination.

ues of the coefficient $O^{(a)}(n, T)=K_{c i}^{(a)}(n, T) / K_{c i}(n, T)=K_{c r}^{(a)}(n, T) / K_{c r}(n, T)$, which describe relative contributions of the associative ionization $(\mathbb{\square}, \mathbb{l})$ are presented in Fig. $\nabla$ by a surface plot. This provides information about abundance of molecular ion $\mathrm{H}_{2}^{+}$. One can see that associative channel is dominant when colliding hydrogen atoms have lower $n$ at chosen temperature (left part of the plot). The importance of associative channel decreases with temperature increase when non-associative channel (마) takes dominant place i.e. destruction of molecular ion.

The obtained results demonstrate the fact that the considered chemi-ionization/ recombination processes are one of the factors of influence on the molecule formation, ionization level and atom excited-state populations and must have some influence on the optical properties of the particular weakly ionized regions in NLS1 galaxies. It's apparent that the importance of these processes for modelling should be investigated. The preliminary results point to further directions of the investigation of the influence of considered processes in NLS1 galaxies as well as to test the impact of (n-n')-mixing processes [ए2]. We expect strong influence of these processes in cooler weakly ionized sufficiently dense parts of BLR clouds in AGNs.

\section{Acknowledgements}

This conference has been organized with the support of the Department of Physics and Astronomy "Galileo Galilei", the University of Padova, the National Institute of Astrophysics INAF, the Padova Planetarium, and the RadioNet consortium. RadioNet has 
received funding from the European Union's Horizon 2020 research and innovation programme under grant agreement No 730562. The authors are thankful to the MESTD of the Republic of Serbia, grants No 176002 and III44002.

\section{References}

[1] M. Crosas and J. C. Weisheit, Hydrogen molecules in quasar broad-line regions, MNRAS 262 (1993) 359.

[2] P. M. Rodríguez-Pascual, J. M. Mas-Hesse, and M. Santos-Lleó, The broad line region of narrow-line Seyfert 1 galaxies, A\&A 327 (1997) 72.

[3] V. Srećković, L. M. Ignjatović and M. S. Dimitrijević, Symmetric atom-atom and ion-atom processes in stellar atmospheres, Atoms 6 (2018).

[4] A. A. Mihajlov, V. A. Srećković, L. M. Ignjatović and A. N. Klyucharev, The Chemi-Ionization Processes in Slow Collisions of Rydberg Atoms with Ground State Atoms: Mechanism and Applications, J. Clust. Sci. 23 (2012) 47.

[5] Y. N. Gnedin, A. A. Mihajlov, L. M. Ignjatović, N. M. Sakan, V. A. Srećković, M. Y. Zakharov et al., Rydberg atoms in astrophysics, New.Astr.Rev. 53 (2009) 259.

[6] Y. N. Gnedin, M. Y. Piotrovich and A. N. Klyucharev, Rydberg States of Atoms and Molecules in the Atmospheres of Very Cool Stars with Magnetic Fields, in Physics and Evolution of Magnetic and Related Stars (Y. Y. Balega, I. I. Romanyuk and D. O. Kudryavtsev, eds.), vol. 494 of ASPCS, p. 261, Apr., 2015.

[7] V. L. Afanas'ev, M. Y. Piotrovich, Y. N. Gnedin, S. D. Buliga and T. M. Natsvlishvili, Spectropolarimetric Observations of Magnetic White Dwarfs with the SAO 6-m Telescope, Astron. Rep. 62 (2018) 138 .

[8] A. A. Mihajlov, L. M. Ignjatović, V. A. Srećković and M. S. Dimitrijević, Chemi-ionization in Solar Photosphere: Influence on the Hydrogen Atom Excited States Population, ApJS 193 (2011) 2.

[9] A. A. Mihajlov, V. A. Srećković, L. M. Ignjatović, A. N. Klyucharev, M. S. Dimitrijević and N. M. Sakan, Non-Elastic Processes in Atom Rydberg-Atom Collisions: Review of State of Art and Problems, J. Astrophys. Astron. 36 (2015) 623 [1611.06333].

[10] A. A. Mihajlov, V. A. Srećković, L. M. Ignjatović and M. S. Dimitrijević, Atom-Rydberg-atom chemi-ionization processes in solar and DB white-dwarf atmospheres in the presence of ( $n$ n')-mixing channels, MNRAS $\mathbf{4 5 8 ( 2 0 1 6 )} 2215$.

[11] A. A. Mihajlov, L. M. Ignjatović, V. A. Srećković and M. S. Dimitrijević, The Influence of Chemi-Ionization and Recombination Processes on Spectral Line Shapes in Stellar Atmospheres, Balt. Astron. 20 (2011) 566.

[12] A. A. Mihajlov, L. M. Ignjatović, V. A. Srećković and Z. Djurić, The influence of $\left(n-n^{\prime}\right)$-mixing processes in $\mathrm{He}^{*}(n)+\mathrm{He}\left(1 \mathrm{~s}^{2}\right)$ collisions on $\mathrm{He}^{*}(n)$ atoms' populations in weakly ionized helium plasmas, एQSRT 109 (2008) 853. 